\title{
Some Applications of the Poisson Process
}

\author{
Kung-Kuen Tse \\ Department of Mathematics, Kean University, Union, USA \\ Email: ktse@kean.edu
}

Received 24 August 2014; revised 20 September 2014; accepted 8 October 2014

Copyright (C) 2014 by author and Scientific Research Publishing Inc.

This work is licensed under the Creative Commons Attribution International License (CC BY).

http://creativecommons.org/licenses/by/4.0/

(c) () Open Access

\section{Abstract}

The Poisson process is a stochastic process that models many real-world phenomena. We present the definition of the Poisson process and discuss some facts as well as some related probability distributions. Finally, we give some new applications of the process.

\section{Keywords}

\section{Poisson Processes, Gamma Distribution, Inter-Arrival Time, Marked Poisson Processes}

\section{Introduction}

Poisson process is used to model the occurrences of events and the time points at which the events occur in a given time interval, such as the occurrence of natural disasters and the arrival times of customers at a service center. It is named after the French mathematician Siméon Poisson (1781-1840). In this paper, we first give the definition of the Poisson process (Section 2). Then we stated some theroems related to the Poisson process (Section 3). Finally, we give some examples and compute the relevant quantities associated with the process (Section 4).

\section{What Is Poisson Process?}

A Poisson process with parameter (rate) $\lambda>0$ is a family of random variables $\left\{N_{t}, t \geq 0\right\}$ satisfying the following properties:

1) $N_{0}=0$.

2) $N_{t_{1}}-N_{t_{0}}, N_{t_{2}}-N_{t_{1}}, \cdots, N_{t_{n}}-N_{t_{n-1}}$ are independent random variables where $0=t_{0}<t_{1}<\cdots<t_{n}$.

3) $P\left(N_{t}-N_{s}=n\right)=\frac{\mathrm{e}^{-\lambda(t-s)}}{n !}(\lambda(t-s))^{n}$ for $t>s$.

$N_{t}=N(0, t]$ can be thought of the number of arrivals up to time $t$ or the number of occurrences up to time $t$. 


\section{Some Facts about the Poisson Process}

We give some properties associated with the Poisson process. The proofs can be found in [1] or [2]. If we let $W_{n}, n \geq 1$ be the time of the $n^{\text {th }}$ arrival $\left(W_{0}=0\right)$, and we let $X_{n+1}=W_{n+1}-W_{n}, n \geq 1$, be the interarrival time $\left(X_{1}=W_{1}\right)$. Then we have the following theorems:

Theorem 1 The $n^{\text {th }}$ arrival time has the $\Gamma$-distribution with density function $f_{W_{n}}(x)=\frac{\lambda(\lambda x)^{n-1}}{(n-1) !} \mathrm{e}^{-\lambda x}$, for $x>0$.

Theorem 2 The interarrival times $X_{1}, X_{2}, \cdots$ are independently exponentially distributed random variables with parameter $\lambda$.

Theorem 3 Conditioned on $N_{t}=n$, the random variables $W_{1}, W_{2}, \cdots, W_{n}$ have the joint density probability function

$$
f_{W_{1}, \cdots, W_{n} \mid N_{t}=n}\left(w_{1}, \cdots, w_{n}\right)=\frac{n !}{t^{n}} \text { for } 0<w_{1}<\cdots<w_{n} \leq t .
$$

Theorem 4 If $Y_{k}$ is a random variable associated with the $k^{\text {th }}$ event in a Poisson process with parameter $\lambda$. We assume that $Y_{1}, Y_{2}, \cdots$ are independent, independent of the Poisson process, and share the common distribution function $G(y)=P\left(Y_{k} \leq y\right)$. The sequence of pairs $\left(W_{1}, Y_{1}\right),\left(W_{2}, Y_{2}\right), \cdots$ is called a marked Poisson process. The $\left(W_{1}, Y_{1}\right),\left(W_{2}, Y_{2}\right), \cdots$ form a two-dimensional nonhomogeneous Poisson point process in the $(t, y)$ plane, where the mean number of points in a region $A$ is given by

$$
\mu(A)=\iint_{A} \lambda g(y) \mathrm{d} y \mathrm{~d} t
$$

The marked Poisson processes have been applied in some geometric probability area [3].

\section{Examples of Poisson Processes}

1) Suppose the number of calls to a phone number is a Poisson process $N_{t}, t \geq 0$ with parameter $\lambda$ and $\tau \sim \exp (\mu)$ is the duration of each call. It is reasonable to assume that $\tau$ is independent of the Poisson process. What is the probability $p$ that the $(n+1)^{\text {st }}$ call gets a busy signal, i.e. it comes when the user is still responding to the $n^{\text {th }}$ call?

For a fixed $\tau$,

$$
\begin{gathered}
P\left(W_{n}+\tau>W_{n+1} \mid \tau\right)=P\left(W_{n+1}-W_{n}<\tau \mid \tau\right)=P\left(X_{n+1}<\tau \mid \tau\right)=\int_{0}^{\tau} \lambda \mathrm{e}^{-\lambda x} \mathrm{~d} x=1-\mathrm{e}^{-\lambda \tau} \\
p=\int_{0}^{\infty} P\left(W_{n}+\tau>W_{n+1} \mid \tau\right) f(\tau) \mathrm{d} \tau=\int_{0}^{\infty} P\left(W_{n}+\tau>W_{n+1} \mid \tau\right) \mu \mathrm{e}^{-\mu \tau} \mathrm{d} \tau \\
=\int_{0}^{\infty}\left(1-\mathrm{e}^{-\lambda \tau}\right) \mu \mathrm{e}^{-\mu \tau} \mathrm{d} \tau=1-\mu \int_{0}^{\infty} \mathrm{e}^{-(\lambda+\mu) \tau} \mathrm{d} \tau=\frac{\lambda}{\lambda+\mu}
\end{gathered}
$$

2) On average, how many calls arrive when the user is on the phone?

Suppose the user is talking on the $n^{\text {th }}$ call,

$$
\begin{gathered}
E\left[N\left(W_{n}, W_{n}+\tau\right] \mid \tau\right]=E[N(0, \tau] \mid \tau]=E\left[N_{\tau} \mid \tau\right]=\lambda \tau \\
E\left[N\left(W_{n}, W_{n}+\tau\right]\right]=E[\lambda \tau]=\lambda E[\tau]=\frac{\lambda}{\mu}
\end{gathered}
$$

3) In a single server system, customers arrive in a bank according to a Poisson process with parameter $\lambda$ and each customer spends $\tau \sim \exp (\mu)$ time with the one and only one bank teller. If the teller is serving a customer, the new customers have to wait in a queue till the teller finishes serving. How long on average does the teller serves the customers up to time $T$ ? (i.e. How long is the server unavailable?)

$$
\begin{aligned}
E\left[\sum_{n=1}^{N_{T}} \tau_{n}\right] & =\sum_{j=0}^{\infty} E\left[\sum_{n=1}^{N_{T}} \tau_{n} \mid N_{T}=j\right] P\left(N_{T}=j\right)=\sum_{j=0}^{\infty} E\left[\sum_{n=1}^{j} \tau_{n} \mid N_{T}=j\right] \frac{\mathrm{e}^{-\lambda T}}{j !}(\lambda T)^{j} \\
& =\sum_{j=0}^{\infty} E\left[\sum_{n=1}^{j} \tau_{n}\right] \frac{\mathrm{e}^{-\lambda T}}{j !}(\lambda T)^{j}=\sum_{j=0}^{\infty} \frac{j}{\mu} \frac{\mathrm{e}^{-\lambda T}}{j !}(\lambda T)^{j}=\frac{\lambda}{\mu} T \mathrm{e}^{-\lambda T} \sum_{j=1}^{\infty} \frac{1}{(j-1) !}(\lambda T)^{j-1}=\frac{\lambda}{\mu} T \mathrm{e}^{-\lambda T} \mathrm{e}^{\lambda T}=\frac{\lambda}{\mu} T
\end{aligned}
$$


4) Suppose team A and team B are engaging in a sport competition. The points scored by team A follows a Poisson process $M_{t}$ with parameter $\lambda$ and the points scored by team B follows a Poisson process $N_{t}$ with parameter $\mu$. Assume that $M_{t}$ and $N_{t}$ are independent, what is the probability that the game ties? Team A wins? Team B wins?

Let $T$ be the duration of the competition.

$$
\begin{gathered}
P(\text { game ties })=P\left(M_{T}=N_{T}\right)=\sum_{k=0}^{\infty} P\left(M_{T}=k, N_{T}=k\right) \\
=\sum_{k=0}^{\infty} P\left(M_{T}=k\right) P\left(N_{T}=k\right)=\sum_{k=0}^{\infty} \frac{\mathrm{e}^{-\lambda t}(\lambda t)^{k}}{k !} \frac{\mathrm{e}^{-\mu t}(\mu t)^{k}}{k !} \\
P(\text { A wins })=P\left(M_{T}>N_{T}\right)=\sum_{k=0}^{\infty} \sum_{\ell=1}^{\infty} P\left(M_{T}=k+\ell, N_{T}=k\right) \\
=\sum_{k=0}^{\infty} \sum_{\ell=1}^{\infty} P\left(M_{T}=k+\ell\right) P\left(N_{T}=k\right)=\sum_{k=0}^{\infty} \sum_{\ell=1}^{\infty} \frac{\mathrm{e}^{-\lambda t}(\lambda t)^{k+\ell}}{(k+\ell) !} \frac{\mathrm{e}^{-\mu t}(\mu t)^{k}}{k !} \\
P(\text { B wins })=P\left(N_{T}>M_{T}\right)=\sum_{k=0}^{\infty} \sum_{\ell=1}^{\infty} P\left(N_{T}=k+\ell, M_{T}=k\right) \\
=\sum_{k=0}^{\infty} \sum_{\ell=1}^{\infty} P\left(N_{T}=k+\ell\right) P\left(M_{T}=k\right)=\sum_{k=0}^{\infty} \sum_{\ell=1}^{\infty} \frac{\mathrm{e}^{-\mu t}(\mu t)^{k+\ell}}{(k+\ell) !} \frac{\mathrm{e}^{-\lambda t}(\lambda t)^{k}}{k !}
\end{gathered}
$$

5) Given that there are $k$ points scored in a match (by both team A and team B), what is the probability that team A scores $\ell$ points, where $\ell \leq k$ ?

$$
\begin{aligned}
& P\left(M_{T}=\ell \mid M_{T}+N_{T}=k\right)=\frac{P\left(M_{T}=\ell, M_{T}+N_{T}=k\right)}{P\left(M_{T}+N_{T}=k\right)} \\
& =\frac{P\left(M_{T}=\ell, N_{T}=k-\ell\right)}{\sum_{j=0}^{k} P\left(M_{T}=j, N_{T}=k-j\right)}=\frac{P\left(M_{T}=\ell\right) P\left(N_{T}=k-\ell\right)}{\sum_{j=0}^{k} P\left(M_{T}=j\right) P\left(N_{T}=k-j\right)} \\
& =\frac{\frac{\mathrm{e}^{-\lambda T}(\lambda T)^{\ell}}{\ell !} \frac{\mathrm{e}^{-\mu T}(\mu T)^{k-\ell}}{(k-\ell) !}}{\sum_{j=0}^{k} \frac{\mathrm{e}^{-\lambda T}(\lambda T)^{j}}{j !} \frac{\mathrm{e}^{-\mu T}(\mu T)^{k-j}}{(k-j) !}}=\frac{\left(\begin{array}{l}
k \\
\ell
\end{array}\right) \lambda^{\ell} \mu^{k-\ell}}{\sum_{j=0}^{k}\left(\begin{array}{l}
k \\
j
\end{array}\right) \lambda^{j} \mu^{k-j}}=\frac{\left(\begin{array}{l}
k \\
\ell
\end{array}\right) \lambda^{\ell} \mu^{k-\ell}}{(\lambda+\mu)^{k}}
\end{aligned}
$$

6) When does a car accident happen? Suppose a street is from west to east and another is from south to north, the two streets intersect at a point $O$. Cars going from west to east arrives at $O$ follows a Poisson process $W_{i}$ with parameter $\lambda$ and cars going from south to east arrives at $O$ follows a Poisson process $\widetilde{W}_{j}$ with parameter $\mu$. It is reasonable to assume that these two processes are independent. If the cars don't slow down and stop at the intersection $O$, then collision happens. The $j^{\text {th }}$ car going from south to north hits the $i^{\text {th }}$ car going from south to east if and only if $W_{i} \leq \widetilde{W}_{j} \leq W_{i}+\tau$, where $\tau$ is the time it takes for the car's tail to reach $O, \tau$ has density function $f_{\tau}(t)$.

$$
\begin{aligned}
P & \left(W_{i} \leq \widetilde{W}_{j} \leq W_{i}+\tau \mid \tau\right)=\int_{0}^{\infty} \int_{x}^{x+\tau} \frac{\lambda(\lambda x)^{i-1} \mathrm{e}^{-\lambda x}}{(i-1) !} \frac{\mu(\mu y)^{j-1} \mathrm{e}^{-\mu y}}{(j-1) !} \mathrm{d} y \mathrm{~d} x \\
& P\left(j^{\text {th }} \text { car from south to north hits } i^{\text {th }} \text { car from west to east }\right) \\
& =\int_{0}^{\infty} P\left(W_{i} \leq \widetilde{W}_{j} \leq W_{i}+\tau \mid \tau\right) f_{\tau}(t) \mathrm{d} t \\
& =\int_{0}^{\infty}\left[\int_{0}^{\infty} \int_{x}^{x+\tau} \frac{\lambda(\lambda x)^{i-1} \mathrm{e}^{-\lambda x}}{(i-1) !} \frac{\mu(\mu y)^{j-1} \mathrm{e}^{-\mu y}}{(j-1) !} \mathrm{d} y \mathrm{~d} x\right] f_{\tau}(t) \mathrm{d} t
\end{aligned}
$$




$$
\begin{aligned}
P(\text { car collision })= & P\left(\bigcup_{i, j} j^{\text {th }} \text { car from south to north hits } i^{\text {th }} \text { car from west to east }\right) \\
& +P\left(\bigcup_{i, j} i^{\text {th }} \text { car from west to east hits } j^{\text {th }} \text { car from south to north }\right) \\
= & \sum_{i, j} \int_{0}^{\infty}\left[\int_{0}^{\infty} \int_{x}^{x+\tau} \frac{\lambda(\lambda x)^{i-1} \mathrm{e}^{-\lambda x}}{(i-1) !} \frac{\mu(\mu y)^{j-1} \mathrm{e}^{-\mu y}}{(j-1) !} \mathrm{d} y \mathrm{~d} x\right] f_{\tau}(t) \mathrm{d} t \\
& +\sum_{i, j} \int_{0}^{\infty}\left[\int_{0}^{\infty} \int_{y}^{y+\tau} \frac{\mu(\mu y)^{j-1} \mathrm{e}^{-\mu y}}{(j-1) !} \frac{\lambda(\lambda x)^{i-1} \mathrm{e}^{-\lambda x}}{(i-1) !} \mathrm{d} x \mathrm{~d} y\right] f_{\tau}(t) \mathrm{d} t
\end{aligned}
$$

7) Occurrences of natural disasters follow a Poisson process with parameter $\lambda$. Suppose that the time it takes to recover and rebuild after the $n^{\text {th }}$ disaster is $Y_{n}$, assume that $Y_{1}, Y_{2}, \cdots$ are independent random variables having the common distribution functions $G(y)=P\left(Y_{k} \leq y\right)$. There are $N_{T}$ disasters up to time $T$, what is the probability that everything is back to normal at time $T$ ? This can also be used as a model for insurance claims. $W_{k}$ is the time for the insurance company to receive the $k^{\text {th }}$ claim and $Y_{k}$ is the time the insurance company takes to settle it. What is the probability that the insurance company is not working on any claim at time $T$ ?

$$
\begin{aligned}
P\left(\max _{1 \leq i \leq N_{T}}\left\{W_{i}+Y_{i}\right\}<T\right) & =\sum_{n=0}^{\infty} P\left(\max _{1 \leq i \leq N_{T}}\left\{W_{i}+Y_{i}\right\}<T \mid N_{T}=n\right) P\left(N_{T}=n\right) \\
& =\sum_{n=0}^{\infty} P\left(\max _{1 \leq i \leq n}\left\{W_{i}+Y_{i}\right\}<T \mid N_{T}=n\right) \frac{\mathrm{e}^{-\lambda T}(\lambda T)^{n}}{n !} \\
& =\sum_{n=0}^{\infty} P\left(W_{1}+Y_{1}<T, \cdots, W_{n}+Y_{n}<T \mid N_{T}=n\right) \frac{\mathrm{e}^{-\lambda T}(\lambda T)^{n}}{n !} \\
& =\sum_{n=0}^{\infty} n ! P\left(U_{1}+Y_{1}<T, \cdots, U_{n}+Y_{n}<T\right) \frac{\mathrm{e}^{-\lambda t}(\lambda T)^{n}}{n !} \\
& =\sum_{n=0}^{\infty} P\left(U_{1}+Y_{1}<T, \cdots, U_{n}+Y_{n}<T\right) \mathrm{e}^{-\lambda t}(\lambda T)^{n}
\end{aligned}
$$

where $U_{1}, \cdots, U_{n}$ are independent and uniformly distributed on $(0, T]$.

$$
\begin{aligned}
P\left(\max _{1 \leq i \leq N_{T}}\left\{W_{i}+Y_{i}\right\}<T\right) & =\sum_{n=0}^{\infty}\left[P\left(U_{1}+Y_{1}<T\right)\right]^{n} \mathrm{e}^{-\lambda T}(\lambda T)^{n} \\
& =\sum_{n=0}^{\infty}\left[\int_{0}^{T} P\left(U_{1}+Y_{1}<T \mid U_{1}=u\right) \frac{1}{T} \mathrm{~d} u\right]^{n} \mathrm{e}^{-\lambda T}(\lambda T)^{n} \\
& =\sum_{n=0}^{\infty}\left[\int_{0}^{T} P\left(Y_{1}<T-u \mid U_{1}=u\right) \frac{1}{T} \mathrm{~d} u\right]^{n} \mathrm{e}^{-\lambda T}(\lambda T)^{n} \\
& =\sum_{n=0}^{\infty}\left[\int_{0}^{T} P\left(Y_{1}<T-u\right) \frac{1}{T} \mathrm{~d} u\right]^{n} \mathrm{e}^{-\lambda T}(\lambda T)^{n} \\
& =\sum_{n=0}^{\infty}\left[\frac{1}{T} \int_{0}^{T} G(T-u) \mathrm{d} u\right]^{n} \mathrm{e}^{-\lambda T}(\lambda T)^{n} \\
& =\mathrm{e}^{-\lambda T} \sum_{n=0}^{\infty}\left[\lambda \int_{0}^{T} G(z) \mathrm{d} z\right]^{n}=\frac{\mathrm{e}^{-\lambda T}}{1-\lambda \int_{0}^{T} G(z) \mathrm{d} z}
\end{aligned}
$$

8) Suppose that $W_{k}$ is the time an insurance company receives the $k^{\text {th }}$ claim and $Y_{k}$ is the time the company takes to settle the claim. What is the average time to settle all claims received before time $T$ ?

The average time to settle all claims received before $T$ is 


$$
E\left[\max _{1 \leq k \leq N_{T}}\left\{W_{k}+Y_{k}\right\}\right]
$$

Suppose $\tau \geq T$,

$$
\begin{aligned}
P\left(\max _{1 \leq k \leq N_{T}}\left\{W_{k}+Y_{k}\right\}<\tau\right) & =\sum_{n=0}^{\infty} P\left(\max _{1 \leq k \leq N_{T}}\left\{W_{k}+Y_{k}\right\}<\tau \mid N_{T}=n\right) P\left(N_{T}=n\right) \\
& =\sum_{n=0}^{\infty} P\left(\max _{1 \leq k \leq n}\left\{W_{k}+Y_{k}\right\}<\tau \mid N_{T}=n\right) \frac{\mathrm{e}^{-\lambda T}}{n !}(\lambda T)^{n} \\
& =\sum_{n=0}^{\infty} P\left(W_{1}+Y_{1}<\tau, \cdots, W_{n}+Y_{n}<\tau \mid N_{T}=n\right) \frac{\mathrm{e}^{-\lambda T}}{n !}(\lambda T)^{n} \\
& =\sum_{n=0}^{\infty} n ! P\left(U_{1}+Y_{1}<\tau, \cdots, U_{n}+Y_{n}<\tau\right) \frac{\mathrm{e}^{-\lambda T}}{n !}(\lambda T)^{n}
\end{aligned}
$$

where $U_{1}, \cdots, U_{n}$ are independent and uniformly distributed on $(0, T]$.

$$
\begin{aligned}
P\left(\max _{1 \leq k \leq N_{T}}\left\{W_{k}+Y_{k}\right\}<\tau\right) & =\sum_{n=0}^{\infty}\left[P\left(U_{1}+Y_{1}<\tau\right)\right]^{n} \mathrm{e}^{-\lambda T}(\lambda T)^{n} \\
& =\sum_{n=0}^{\infty}\left[\int_{0}^{T} P\left(U_{1}+Y_{1}<\tau \mid U_{1}=u\right) \frac{1}{T} \mathrm{~d} u\right]^{n} \mathrm{e}^{-\lambda T}(\lambda T)^{n} \\
& =\sum_{n=0}^{\infty}\left[\int_{0}^{T} P\left(Y_{1}<\tau-u \mid U_{1}=u\right) \frac{1}{T} \mathrm{~d} u\right]^{n} \mathrm{e}^{-\lambda T}(\lambda T)^{n} \\
& =\sum_{n=0}^{\infty}\left[\frac{1}{T} \int_{0}^{T} P\left(Y_{1}<\tau-u\right) \mathrm{d} u\right]^{n} \mathrm{e}^{-\lambda T}(\lambda T)^{n} \\
& =\sum_{n=0}^{\infty}\left[\frac{1}{T} \int_{0}^{T} G(\tau-u) \mathrm{d} u\right]^{n} \mathrm{e}^{-\lambda T}(\lambda T)^{n} \\
& =\sum_{n=0}^{\infty}\left[\frac{1}{T} \int_{\tau-T}^{\tau} G(z) \mathrm{d} z\right]^{n} \mathrm{e}^{-\lambda T}(\lambda T)^{n} \\
& =\mathrm{e}^{-\lambda T} \sum_{n=0}^{\infty}\left[\lambda \int_{\tau-T}^{\tau} G(z) \mathrm{d} z\right]^{n}=\frac{\mathrm{e}^{-\lambda T}}{1-\lambda \int_{\tau-T}^{\tau} G(z) \mathrm{d} z}
\end{aligned}
$$

Clearly, $P\left(\max _{1 \leq k \leq N_{T}}\left\{W_{k}+Y_{k}\right\}<\tau\right)=0$ for $\tau<T$.

$$
\begin{aligned}
E\left[\max _{1 \leq k \leq N_{T}}\left\{W_{k}+Y_{k}\right\}\right] & =\int_{0}^{\infty} P\left(\max _{1 \leq k \leq N_{T}}\left\{W_{k}+Y_{k}\right\}>\tau\right) \mathrm{d} \tau \\
& =T+\int_{T}^{\infty} P\left(\max _{1 \leq k \leq N_{T}}\left\{W_{k}+Y_{k}\right\}>\tau\right) \mathrm{d} \tau \\
& =T+\int_{T}^{\infty}\left(1-\frac{\mathrm{e}^{-\lambda T}}{1-\lambda \int_{\tau-T}^{\tau} G(z) \mathrm{d} z}\right) \mathrm{d} \tau
\end{aligned}
$$

9) Customers arrive at a shopping mall follows a Poisson process with parameter $\lambda$. The time the customers spend in the store $Y_{1}, Y_{2}, \cdots$ are independent random variables having the common distribution function $G(y)=P\left(Y_{k} \leq y\right)$. Let $M_{t}$ be the number of customers exist up to the closing time $t$. What is the expected number of customers in the mall at time $t$ ?

Condition on $N_{t}=n$ and let $W_{1}, \cdots, W_{n} \leq t$ be the arrival time of the customers. Then customer $k$ exists in the mall at time $t$ if and only if $W_{k}+Y_{k} \geq t$. Let the random variable

$$
\mathbb{I}\left\{W_{k}+Y_{k} \geq t\right\}= \begin{cases}1 & \text { if } W_{k}+Y_{k} \geq t, \\ 0 & \text { if } W_{k}+Y_{k}<t .\end{cases}
$$


Then $\mathbb{I}\left\{W_{k}+Y_{k} \geq t\right\}=1$ if and only if the $k^{\text {th }}$ customer exists in the mall at time $t$. Thus

$$
\begin{aligned}
P\left(M_{t}=m \mid N_{t}=n\right) & =P\left(\sum_{k=1}^{N_{t}} \mathbb{I}\left\{W_{k}+Y_{k} \geq t\right\}=m \mid N_{t}=n\right) \\
& =P\left(\sum_{k=1}^{n} \mathbb{I}\left\{W_{k}+Y_{k} \geq t\right\}=m \mid N_{t}=n\right)=P\left(\sum_{k=1}^{n} \mathbb{I}\left\{U_{k}+Y_{k} \geq t\right\}=m\right)
\end{aligned}
$$

where $U_{1}, U_{2}, \cdots, U_{n}$ are independent and uniformly distributed on $(0, t] . P\left(\sum_{k=1}^{n} \mathbb{I}\left\{U_{k}+Y_{k} \geq t\right\}=m\right)$ is the binomial distribution in which

$$
\begin{aligned}
p & =P\left(U_{k}+Y_{k} \geq t\right)=\int_{0}^{t} P\left(U_{k}+Y_{k} \geq t \mid U_{k}=u\right) \frac{1}{t} \mathrm{~d} u \\
& =\int_{0}^{t} P\left(Y_{k} \geq t-u \mid U_{k}=u\right) \frac{1}{t} \mathrm{~d} u=\frac{1}{t} \int_{0}^{t} P\left(Y_{k} \geq t-u\right) \mathrm{d} u \\
& =\frac{1}{t} \int_{0}^{t}[1-G(t-u)] \mathrm{d} u=\frac{1}{t} \int_{0}^{t}[1-G(z)] \mathrm{d} z
\end{aligned}
$$

Hence,

$$
\begin{aligned}
P\left(M_{t}=m\right) & =\sum_{n=m}^{\infty} P\left(M_{t}=m \mid N_{t}=n\right) P\left(N_{t}=n\right) \\
& =\sum_{n=m}^{\infty} \frac{n !}{m !(n-m) !} p^{m}(1-p)^{n-m} \frac{(\lambda t)^{n} \mathrm{e}^{-\lambda t}}{n !} \\
& =\mathrm{e}^{-\lambda t} \frac{(\lambda p t)^{m}}{m !} \sum_{n=m}^{\infty} \frac{(1-p)^{n-m}(\lambda t)^{n-m}}{(n-m) !} \\
& =\mathrm{e}^{-\lambda t} \frac{(\lambda p t)^{m}}{m !} \mathrm{e}^{\lambda t(1-p)}=\frac{\mathrm{e}^{-\lambda p t}(\lambda p t)^{m}}{m !}
\end{aligned}
$$

That is, the number of customers existing at time $t$ has a Poisson distribution with mean

$$
\lambda p t=\lambda \int_{0}^{t}[1-G(y)] \mathrm{d} y .
$$

The average number of customers exist at the mall closing time is

$$
E\left[M_{t}\right]=\frac{1}{\lambda \int_{0}^{t}[1-G(y)] \mathrm{d} y} .
$$

10) Customers arriving at a service counter follows a Poisson process with parameter $\lambda$. Let $M_{t}$ be the number of customers served longer than $\tau$ up to time $t$. What is the distribution of $M_{t}$ ?

Condition on $N_{t}=n$ and let $W_{1}, \cdots, W_{n} \leq t$ be the arrival time of the customers. Let the random variable

$$
\mathbb{I}\left\{Y_{k}>\tau\right\}= \begin{cases}1 & \text { if } Y_{k}>\tau \\ 0 & \text { if } Y_{k} \leq \tau .\end{cases}
$$

Then $\mathbb{I}\left\{Y_{k}>\tau\right\}=1$ if and only if the $k^{\text {th }}$ customer served longer than $\tau$. Thus

$$
\begin{aligned}
P\left(M_{t}=m \mid N_{t}=n\right) & =P\left(\sum_{k=1}^{N_{t}} \mathbb{I}\left\{Y_{k}>\tau\right\}=m \mid N_{t}=n\right) \\
& =P\left(\sum_{k=1}^{n} \mathbb{I}\left\{Y_{k}>\tau\right\}=m \mid N_{t}=n\right) \\
& =P\left(\sum_{k=1}^{n} \mathbb{I}\left\{Y_{k}>\tau\right\}=m\right),
\end{aligned}
$$


which is the binomial distribution with $p=P\left(Y_{k}>\tau\right)=1-G(\tau)$. Hence,

$$
\begin{aligned}
P\left(M_{t}=m\right) & =\sum_{n=m}^{\infty} P\left(M_{t}=m \mid N_{t}=n\right) P\left(N_{t}=n\right) \\
& =\sum_{n=m}^{\infty} \frac{n !}{m !(n-m) !} p^{m}(1-p)^{n-m} \frac{(\lambda t)^{n} \mathrm{e}^{-\lambda t}}{n !} \\
& =\mathrm{e}^{-\lambda t} \frac{(\lambda p t)^{m}}{m !} \sum_{n=m}^{\infty} \frac{(1-p)^{n-m}(\lambda t)^{n-m}}{(n-m) !} \\
& =\mathrm{e}^{-\lambda t} \frac{(\lambda p t)^{m}}{m !} \mathrm{e}^{\lambda t(1-p)}=\frac{\mathrm{e}^{-\lambda p t}(\lambda p t)^{m}}{m !}
\end{aligned}
$$

That is, the number of customers served longer than $\tau$ has a Poisson distribution with mean

$$
\lambda p t=\lambda(1-G(\tau)) t .
$$

\section{Conclusion}

Poisson process is one of the most important tools to model the natural phenomenon. Some important distributions arise from the Poisson process: the Poisson distribution, the exponential distribution and the Gamma distribution. It is also used to build other sophisticated random process.

\section{References}

[1] Taylor, H.M. and Karlin, S. (1998) An Introduction to Stochastic Modeling. Academic Press, Waltham.

[2] Ross, S.M. (1993) Introduction to Probability Models. 5th Edition, Academic Press, Waltham.

[3] Penrose, M.D. (2000) Central Limit Theorems for $k$-Nearest Neighbor Distances. Stochastic Processes and their Applications, 85, 295-320. http://dx.doi.org/10.1016/S0304-4149(99)00080-0 
Scientific Research Publishing (SCIRP) is one of the largest Open Access journal publishers. It is currently publishing more than 200 open access, online, peer-reviewed journals covering a wide range of academic disciplines. SCIRP serves the worldwide academic communities and contributes to the progress and application of science with its publication.

Other selected journals from SCIRP are listed as below. Submit your manuscript to us via either submit@scirp.org or Online Submission Portal.
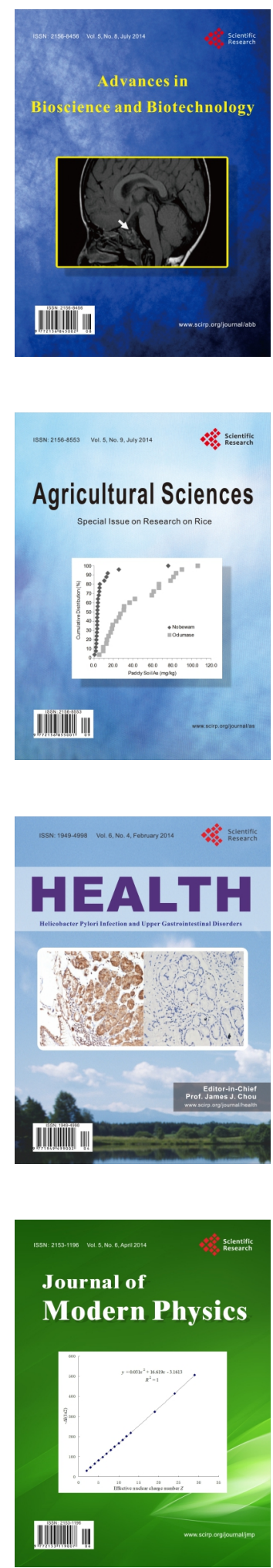
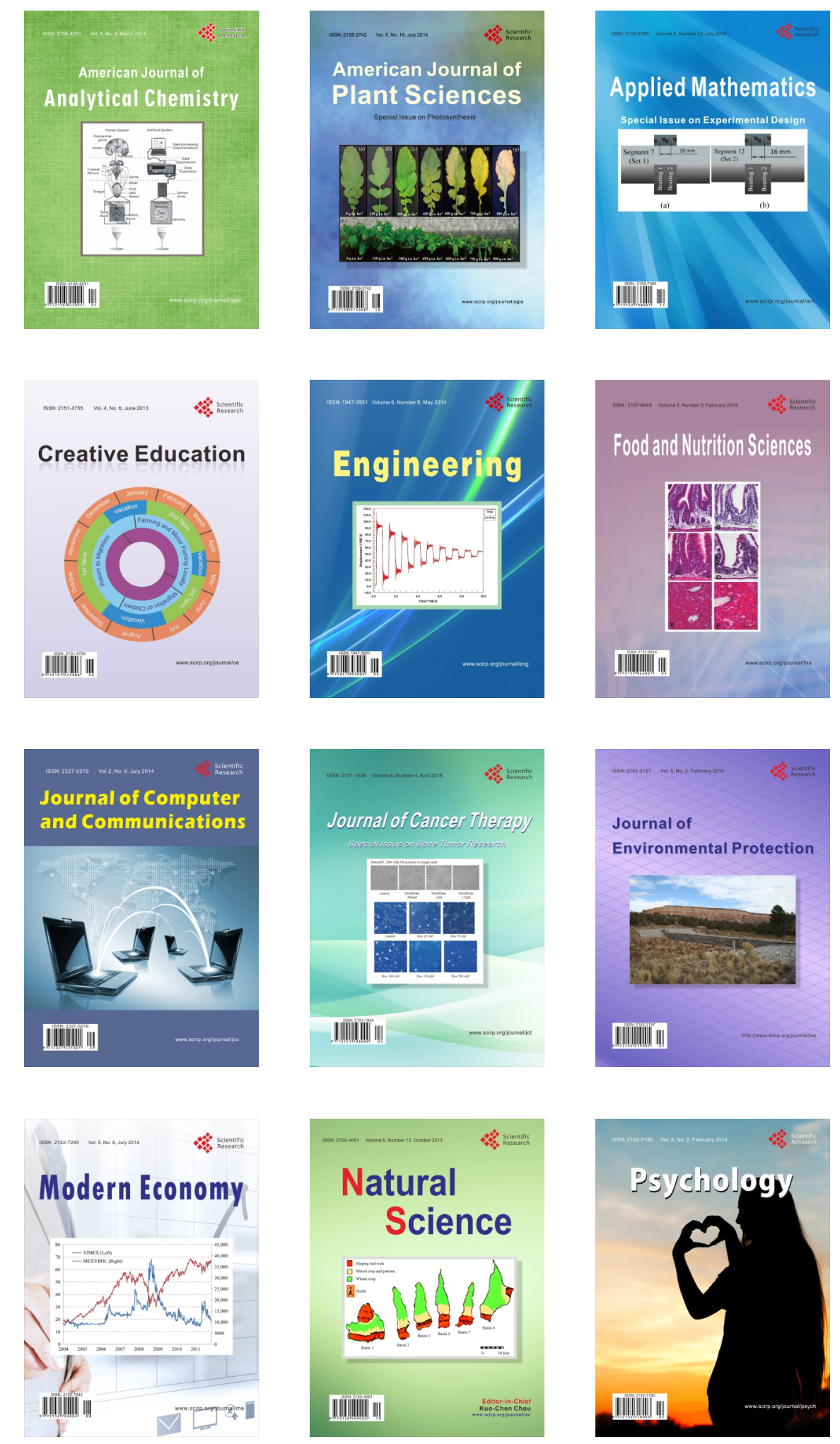